\title{
The influence of the negative wake on the deformation and breakup of viscoelastic droplets
}

\author{
Fernando Carril-Naranjo ${ }^{1}$, Baltsar Mena, Didier Samayoa ${ }^{2}$, Antonio Guerrero ${ }^{3}$ and Bernardo Figueroa-Espinoza ${ }^{1, *}$ \\ ${ }^{1}$ Universidad Nacional Autónoma de México, Instituto de Ingeniería, LIPC, Yucatán, Sisal 97355, Mexico \\ ${ }^{2}$ Instituto Politécnico Nacional, SEPI-ESIME, Unidad Profesional Adolfo López Mateos, Mexico City 07738, Mexico \\ ${ }^{3}$ Escuela Politécnica Superior, Virgen de Africa 7, Sevilla 41011, Spain
}

(Received October 7, 2020; final revision received April 15, 2021; accepted June 8, 2021)

\begin{abstract}
Experiments were performed using visual and PIV techniques in order to study the appearance of a negative wake as well as its influence upon the deformation and breakup of droplets rising in viscoelastic fluids. In this report, Newtonian and viscoelastic drops were injected through different viscoelastic fluids; the deformation of the droplets was then followed and analyzed. In the case of Newtonian drops traveling through a viscoelastic fluid, a tail appears which later breaks into satellite droplets; a negative wake is present on the sides of the tail. The viscoelastic drops also exhibit a tail which is more resistant to rupture and the negative wake appears after the tail; additionally, a bump appears at the tip of the tail which enhances its elongation and determines the onset of breakup.
\end{abstract}

Keywords: viscoelastic droplets, negative wake, PIV, tail breakup

\section{Introduction}

The deformation and breakup of droplets moving in Newtonian and non-Newtonian fluids is a subject of importance in various topics, i.e. the flow of blood clots in veins and arteries (Campo-Deaño et al., 2013), multiphase flows, oil recovery processes (Hou et al., 2005) and emulsions (Matsumura et al., 1993), among many others. Droplet stability and breakup is crucial in the study of aerosol distribution, including virus transmission through saliva (Plog et al., 2020). Moreover, the importance of viscoelastic drops and composites cannot be understated in the field of polymers, paints and coatings (Brinson and Brinson, 2008), and new materials science (Suhr et al., 2005).

The deformation of droplets has been studied extensively by many authors (see for example the reviews by Caswell et al. (2004) and Zenit and Feng (2018)). Also, particular attention has been given to flow conditions of simple shear and elongational flows, where the uncommon phenomenon of negative wake is observed (Hassager, 1979; Cherdhirankorn, 2004). In order to understand these flows, Milliken and Leal developed a four-roll mill which allows for a controlled elongational flow (Milliken and Leal, 1991). Cherdhirankon et al. (2004) studied the deformation of viscoelastic droplets in a viscoelastic external flow, and Mukerje et al. (2009) analyzed the deformation of a viscoelastic drop in a Newtonian matrix under simple shear flow. Given the difficulty of carrying out measurements of important variables (such as shear

*Corresponding author; E-mail: bfigueroae@iingen.unam.mx stress) on deformable droplets, a considerable amount of work has been carried out using numerical simulations to study negative wakes (Bush, 1993; Satrape and Crochet, 1994; Joseph and Feng, 1995; Harlen, 2002; Dou and Phan-Thien, 2003; Frank and Li, 2006; Mendoza-Fuentes et al., 2009); both experimental and numerical results suggest that the elastic properties of the fluids are responsible for the negative wake onset.

Nevertheless, there is not enough experimental data on the deformation mechanism of viscoelastic drops rising freely through a viscoelastic fluid. More recently, Ortiz et al. (2016) have found that when a viscoelastic drop travels through a non-Newtonian fluid the droplet is deformed and a negative wake appears. This negative wake tends to stretch the drop by creating a region of uniaxial extension. Although it is true that the negative wake phenomena is well documented for solid-spheres (Broadbent and Mena, 1974; Manero and Mena, 1981; Bisgaard, 1983; Mena et al., 1987; Arigo and Mckinley, 1998; Frank and Li, 2006) and for bubbles (Hassager, 1979; Kemiha et al., 2006; Soto et al., 2006; Imaizumi et al., 2014; Fraggedakis et $a l ., 2016$ ), the interaction between deformable droplets and negative wakes is still unclear (Ortiz et al., 2016).

The aim of this work is to study the influence of the negative wake upon the deformation of a droplet as it rises through different non-Newtonian fluids.

\section{Experimental Setup}

The experimental arrangement consists of an acrylic container of square cross section $150 \mathrm{~mm}$ in width and $800 \mathrm{~mm}$ height. The container houses the fluid through which the droplets travel. The droplets were injected indi- 


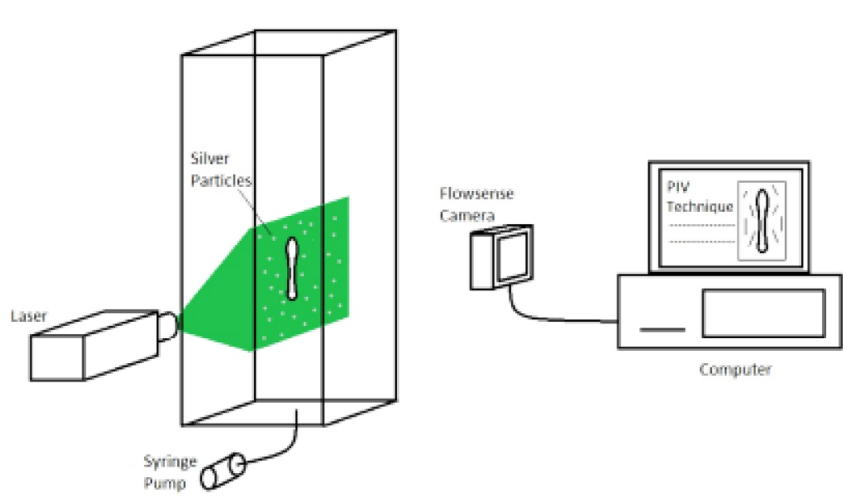

Fig. 1. (Color online) Experimental Setup. A laser sheet generated with an Evergreen Laser cuts the middle plane of the droplet. Images were taken with a frequency of $15 \mathrm{~Hz}$ with a Flow Sense EO M4 camera. The images were analyzed using a crosscorrelation algorithm.

vidually through an orifice at the bottom of the container with a $5 \mathrm{ml}$ syringe pump. Their volume varied from 1 to $5 \mathrm{ml}$.

In order to study the influence of the negative wake on the droplet deformation, a PIV system was used (Dantec Dynamics $^{\mathrm{TM}}$ ). A laser sheet was generated with an Evergreen Laser located parallel to the container, cutting through the middle plane of the drop. The external fluid was inseminated using silver Dantec Dynamics particles of $10 \mu \mathrm{m}$ as seeding particles. The images were taken with a Flow Sense ${ }^{\mathrm{TM}}$ EO M4 camera with a resolution of 2048 $\times 2048$. The camera was placed perpendicular to the laser sheet at $10 \mathrm{~cm}$ over the bottom of the container. At this point the drop travels at its terminal rising velocity but still keeping its original shape. The images were analyzed with a cross-correlation algorithm which yielded the flow field and the final rising velocity of the droplets (details may be found in Carril (2019)). A general layout of the experimental setup is shown in Fig. 1.

\section{Experimental Fluids}

The Newtonian droplets were made of commercial maple honey and of motor oils of various viscosities. In particular, a SAE 80W-90 Oil was used. For the viscoelastic droplets, a Poly-isobutylene $(0.5 \%$ per weight $)$ in a mixture of $51.3 \%$ polybutene oil and $48.7 \%$ decalin was used. This fluid has been extensively used in the literature and was the subject of intensive study several decades ago (see Volume 52 in 1994 of "Journal of Non-Newtonian Fluid Mechanics"). The rheological properties of the external fluid were controlled by changing the polymer concentration in a distilled water solution; two different concentrations of poly-acrylamide Separan AP-30 in distilled water were used (3\% per weight and 5\% per weight). These solutions have been extensively used by our group for many years. The physical properties of the fluids are listed in Table 1.

The rheological measurements were performed using a LS-ARES TA Instrument rheometer. A $6 \mathrm{~mm} \varnothing$ coneplate configuration was used for the oscillatory tests at 25 degrees Celsius. The relaxation time of the viscoelastic fluids was obtained from measurements of rheological properties i.e. normal force, shear stress, shear rate (coneplate and parallel plates rheometry were used). Relaxation time can be obtained from the intersection of $G^{\prime}$ and $G^{\prime \prime}$ in oscillatory measurements and from normal force measurements (N1) and viscosity measurements at low shear rates in viscometric flows using an appropriate constitutive equation such as an Oldroyd B. These experimental fluid properties were previously reported in Manero and Mena (1981) and Ortiz et al. (2016). The maple honey and the engine oil have constant viscosities; honey with $\eta \approx 0.25$ $\mathrm{Pa} \cdot \mathrm{s}$ and the oil with $\eta \approx 0.02 \mathrm{~Pa} \cdot \mathrm{s}$. The non-Newtonian fluids showed a viscoelastic shear-thinning behavior. The results are summarized in Figs. 2, 3, and 4. Surface tension measurements were performed using a Du Nouy ring with pendant drop method. Surface tension between the $\mathrm{S} 1$ droplet and the surrounding fluids was very similar for both concentrations $(58 \mathrm{mN} / \mathrm{m})$. The capillary number varied between 0.15 and 0.2 and in every case the Weber number was close to 0.01 . Therefore, the effect of surface tension was kept as constant as possible whilst inertia effects were negligible since the density difference between the droplet and the outer fluid was very small; so was the rising velocity of the droplet (less than $1 \mathrm{~mm} / \mathrm{s}$ ) and therefore the characteristic shear rate (around $0.1 \mathrm{~s}^{-1}$ ). In summary, the dominating forces in the experiments are mainly viscous and elastic. Therefore, the dimensionless numbers to be considered are the Reynolds number $R e$

Table 1. Properties and composition of experimental fluids.

\begin{tabular}{cccc}
\hline \hline Number & Formulation & Density $\left(\mathrm{g} / \mathrm{cm}^{3}\right)$ & Relaxation time $(\mathrm{s})$ \\
\hline 1 & Poly-Isobutylene (0.5\%) dissolved in a mixture of polybutene oil & 0.903 & 13.09 \\
2 & $(51.3 \%)$ and decalin (48.7). This fluid is known as S1A. & 0.992 & 0.097 \\
3 & Polyacrylamide (3\% Separan AP-30) in water & 0.997 & 0.142 \\
4 & Polyacrylamide (5\% Separan AP-30) in water & 0.962 & - \\
5 & SAE 80W-90 Oil & 1.31 & - \\
\hline
\end{tabular}


The influence of the negative wake on the deformation and breakup of viscoelastic droplets

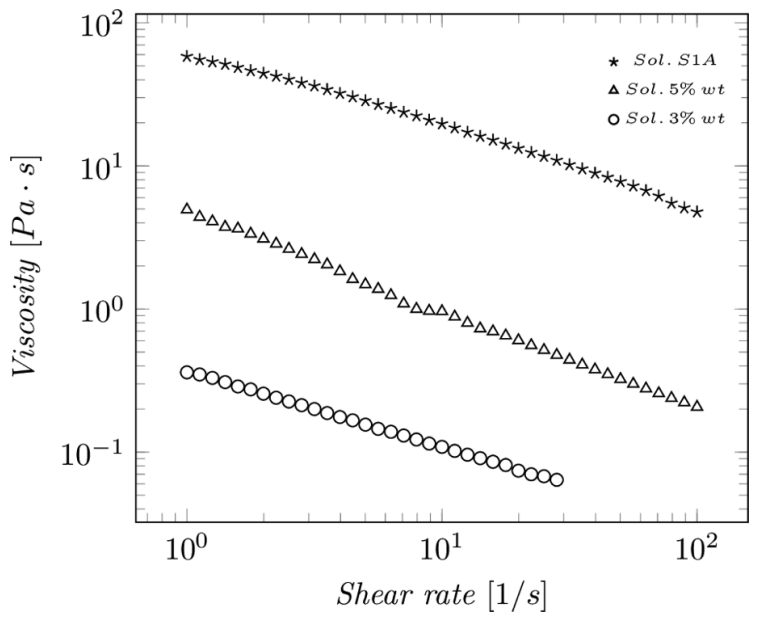

Fig. 2. Rheological measurements for the viscoelastic fluids.

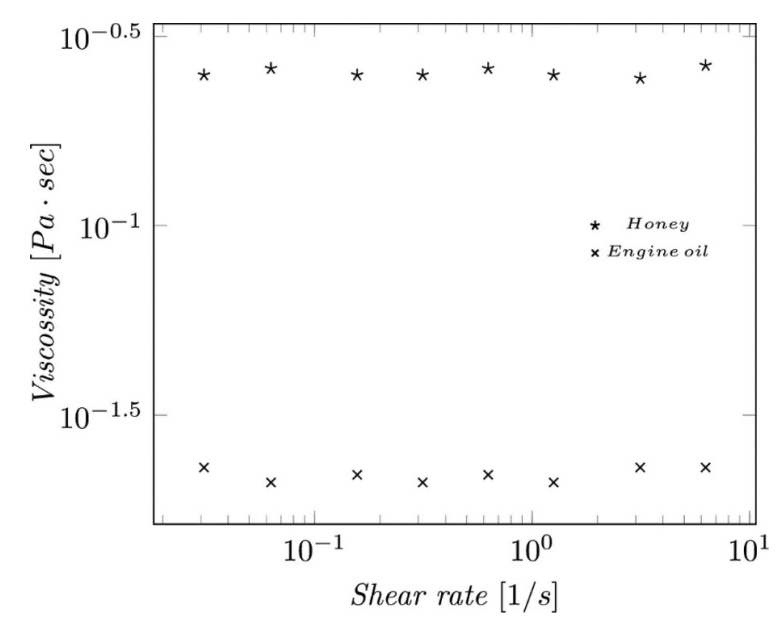

Fig. 3. Newtonian fluid properties.

and the Weissenberg, Wi.

Figures 2 and 3 show the viscosity of the Newtonian and viscoelastic fluids used in the experiments, respectively.

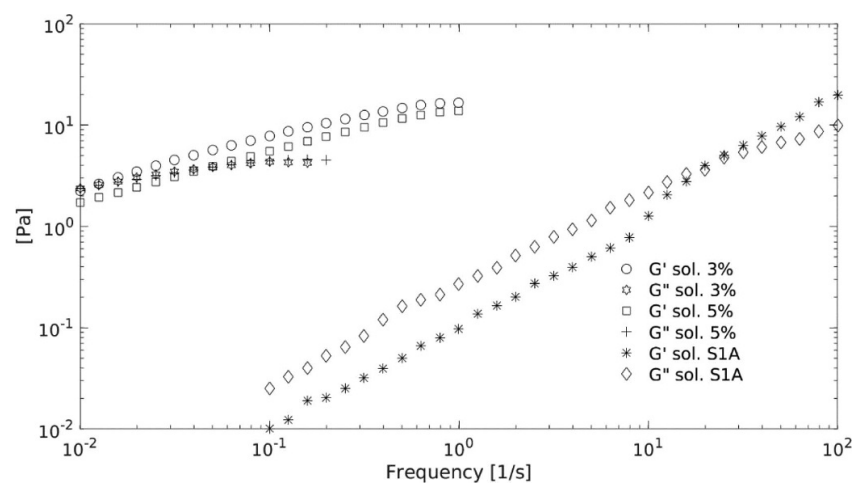

Fig. 4. Storage modulus $G^{\prime}$ and Loss modulus $G^{\prime \prime}$ as a function of frequency, for the viscoelastic solutions.

Figure 4 summarizes the Storage and Loss moduli of the viscoelastic fluids from oscillatory tests, in terms of frequency.

\section{Experimental Results}

Several different situations were examined, namely: 4.1. Newtonian droplets rising in a viscoelastic fluid 4.2. viscoelastic droplets rising in a Newtonian fluid 4.3. viscoelastic droplets rising in a viscoelastic fluid Table 2 summarizes the obtained experimental data. All tests correspond to very low Reynolds number, and the viscoelastic properties of the inner and outer fluids are represented by the Relaxation times and Weissenberg number ( $W i$ and $R e$ defined in the header of the table, where $\lambda$ is the relaxation time, $U$ the terminal rise velocity, $\mu$ the dynamic viscosity, $\rho$ the density and $L$ the droplet diameter).

\subsection{Newtonian droplets rising in a non-Newtonian fluid}

Table 2. Experimental conditions for the droplets and outer fluid.

\begin{tabular}{cccccccccc}
\hline \hline $\begin{array}{c}\text { Droplet } \\
\text { fluid }\end{array}$ & Outer fluid & $\begin{array}{c}\text { Droplet } \\
\text { volume } \\
(\mathrm{ml})\end{array}$ & $\begin{array}{c}\text { Velocity } \\
(\mathrm{m} / \mathrm{s})\end{array}$ & $\begin{array}{c}\text { Outer } \\
\text { Density } \\
\left(\mathrm{g} / \mathrm{cm}^{3}\right)\end{array}$ & $\begin{array}{c}\text { Droplet } \\
\text { diameter } \\
(\mathrm{m})\end{array}$ & $\begin{array}{c}\text { Outer fluid } \\
\text { viscosity } \\
(\text { Pa.s })\end{array}$ & $\begin{array}{c}\text { Droplet } \\
\text { Relaxation time } \\
(\mathrm{s})\end{array}$ & $\begin{array}{c}\text { Re } \\
\frac{L U \rho}{\mu}\end{array}$ & $\begin{array}{c}\text { Wi } \\
\frac{\lambda U}{L}\end{array}$ \\
\hline S1A & 5\%wt mixture & 1 & 0.0005 & 992 & 0.0112 & 4.945 & 13.09 & 0.00108 & 0.5570 \\
S1A & 5\%wt mixture & 3 & 0.0015 & 992 & 0.0119 & 4.945 & 13.09 & 0.00358 & 1.6472 \\
S1A & 5\%wt mixture & 5 & 0.0017 & 992 & 0.0123 & 4.945 & 13.09 & 0.00425 & 1.8275 \\
S1A & 3\%wt mixture & 1 & 0.0107 & 997 & 0.0107 & 1.094 & 13.09 & 0.10453 & 13.09 \\
S1A & 3\%wt mixture & 3 & 0.0128 & 997 & 0.0158 & 1.094 & 13.09 & 0.18561 & 10.612 \\
S1A & 3\%wt mixture & 5 & 0.0173 & 997 & 0.0168 & 1.094 & 13.09 & 0.26581 & 13.447 \\
Oil & 5\%wt mixture & 1 & 0.0002 & 992 & 0.0102 & 4.945 & 0 & 0.00040 & 0 \\
Oil & 5\%wt mixture & 3 & 0.0012 & 992 & 0.0114 & 4.945 & 0 & 0.00274 & 0 \\
Oil & 5\%wt mixture & 5 & 0.0024 & 992 & 0.0143 & 4.945 & 0 & 0.00694 & 0 \\
Oil & 3\%wt mixture & 1 & 0.0121 & 997 & 0.0101 & 1.094 & 0 & 0.11089 & 0 \\
Oil & 3\%wt mixture & 3 & 0.0192 & 997 & 0.0108 & 1.094 & 0 & 0.18735 & 0 \\
Oil & 3\%wt mixture & 5 & 0.0240 & 997 & 0.0114 & 1.094 & 0 & 0.24977 & 0 \\
\hline
\end{tabular}




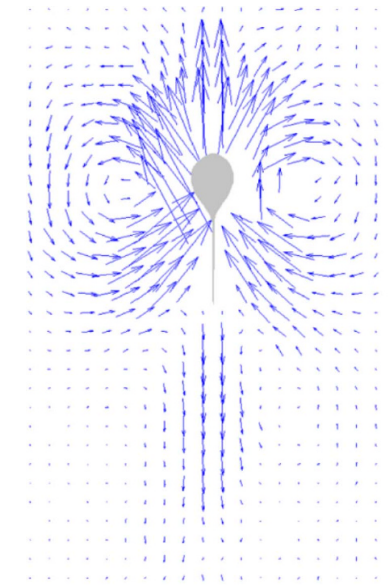

(a)

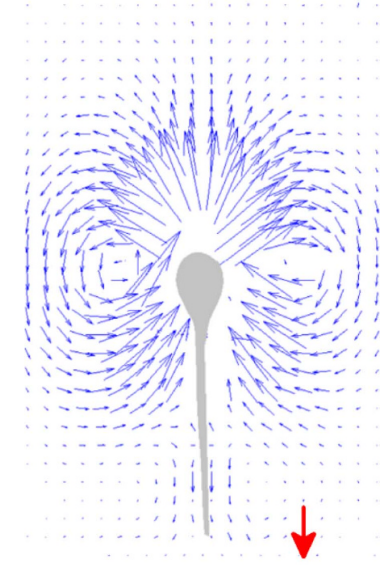

(b)
Fig. 5. (Color online) Flow field around Newtonian (a) $1 \mathrm{ml}$ and (b) $5 \mathrm{ml}$ drops (oil engine) in 3\% wt Separan solution. The rising velocity was $0.12 \times 10^{-2} \mathrm{~m} / \mathrm{s}$ with $R=0.1 \times 10^{-1}$ (a) and $0.24 \times 10^{-2}$ $\mathrm{m} / \mathrm{s}$ with $R=0.25 \times 10^{-1}$ for (b). The drops' rising velocity increases as the volume of the drop increases. The red arrow represents $0.1 \times 10^{-2} \mathrm{~m} / \mathrm{s}$, and the same scale is used in subsequent figures for PIV vector fields.

Newtonian drops injected in the non-Newtonian fluids adopted a tear-like shape with a tail. For small droplets below $2 \mathrm{ml}$ volume, a slim and short tail appeared (Fig. $5 \mathrm{a})$. The droplets over $2 \mathrm{ml}$ volume presented a thick and long tail which breaks into large drops (Fig. 5b). In all of these cases, a negative wake was present in the flow profile. Note that the negative wake may appear above the tip of the tail, which contributes to the formation of an elongational flow which does not affect the shape of the Newtonian drop (Fig. 5b). The red arrow in the bottom of the image represents a velocity of $0.1 \times 10^{-2} \mathrm{~m} / \mathrm{s}$.

\subsection{Viscoelastic droplets rising in a Newtonian fluid}

The initial shape of the injected droplet was spherical, and evolved into a tear-like shape with a tail that resulted resistant to rupture. This behavior was observed for droplet volumes from 1 to $5 \mathrm{ml}$. The flow profile consists of an upward flow around the head and tail (Fig. 6). Sometimes, the droplet presented an oscillatory rising path, which complicated the PIV measurements. No negative wake appears since the outer fluid is Newtonian.

\subsection{Viscoelastic droplets rising through viscoelastic media}

In order to examine the effect of different elastic and viscous properties of the surrounding fluid upon the deformation of the rising droplet as well as regarding the position of the negative wake in the flow, two different polymer concentrations were considered. A dilute 3\% Separan solution and a more concentrated 5\% solution were used. The same polymer was used in order to pre-

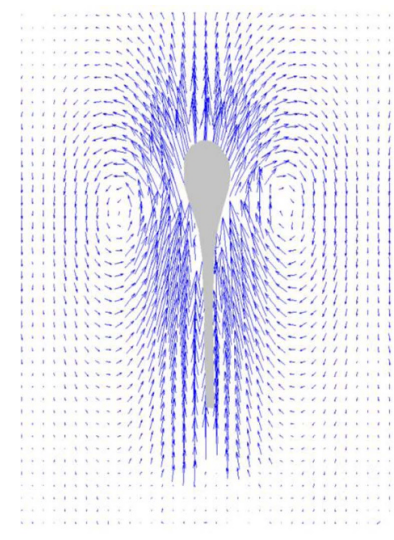

(a)

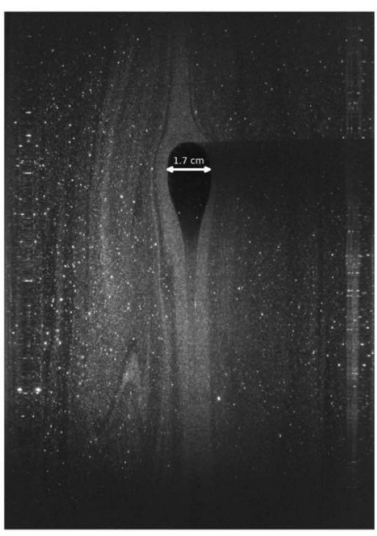

(b)
Fig. 6. (Color online) (a) Flow field around viscoelastic $5 \mathrm{ml}$ drop in Newtonian fluid and (b) PIV image before cross correlation analysis. The maximum cross-section diameter of the drop is $1.7 \mathrm{~cm}$ (see white arrow in (b)). A similar flow field appears for all droplets from $1 \mathrm{ml}$ to $5 \mathrm{ml}$ rising through a Newtonian fluid; and upward flow around the head and tail of the droplet. This shows that a viscoelastic drop may deform with no negative wake.

serve the rheological properties other than the viscous and elastic characteristics. The viscosity was increased approximately 5 times and the relaxation time by two, while the Webber number and the Capillary number remained virtually the same.

\subsubsection{3\% Separan solution}

The viscoelastic drops injected through a dilute 3\%wt Separan solution presented two different behaviors depending on their volume. Drops smaller than $2(\mathrm{ml})$ showed tear-like shape and kept this shape all the way up. An example of the flow field of this type of drops is

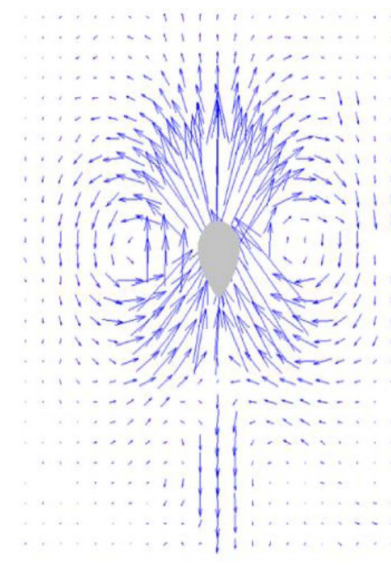

(a)

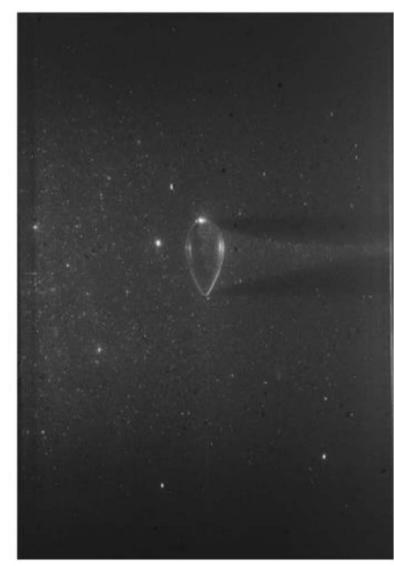

(b)
Fig. 7. (Color online) (a) Flow field around a viscoelastic $1 \mathrm{ml}$ drop in 3\% wt Separan solution and (b) PIV image before cross correlation analysis. The drop keeps the tear-like shape all the way up despite the presence of the negative wake. 
The influence of the negative wake on the deformation and breakup of viscoelastic droplets

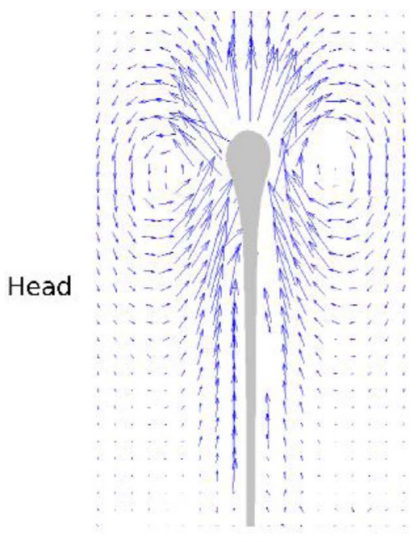

(a)

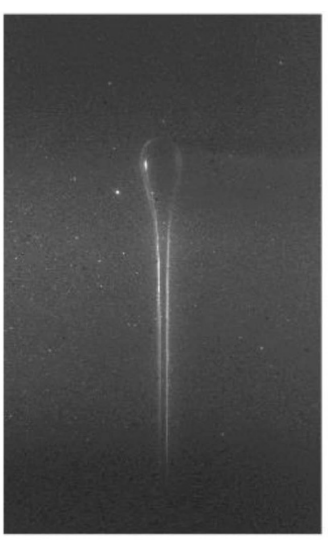

(b)

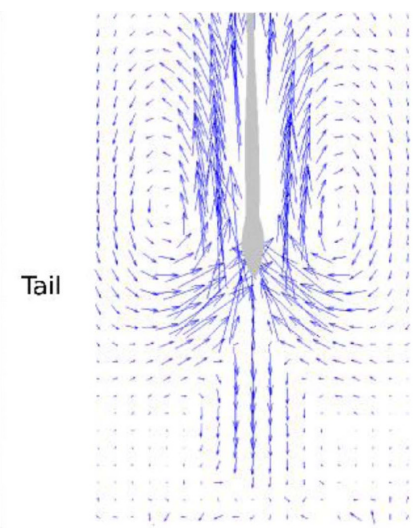

(c)

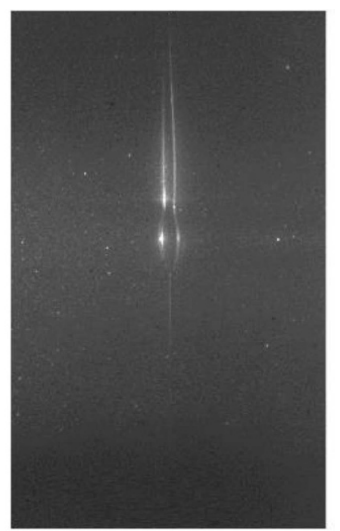

(d)

Fig. 8. (Color online) Viscoelastic $3 \mathrm{ml}$ drop in 3\% wt Separan solution. The drop is divided into two sections, head and tail. The head shows a sphere flow field followed by an upward flow. The tail shows an upward flow. After the tail a negative wake appears.

shown in Fig. 7.

During the ascent, droplets over $2 \mathrm{ml}$ volume showed a deformed shape. These drops initially had a tear-like shape and later developed a flat tail, which stretched until breaking into drops of different volumes. These droplets are able to stretch considerably.

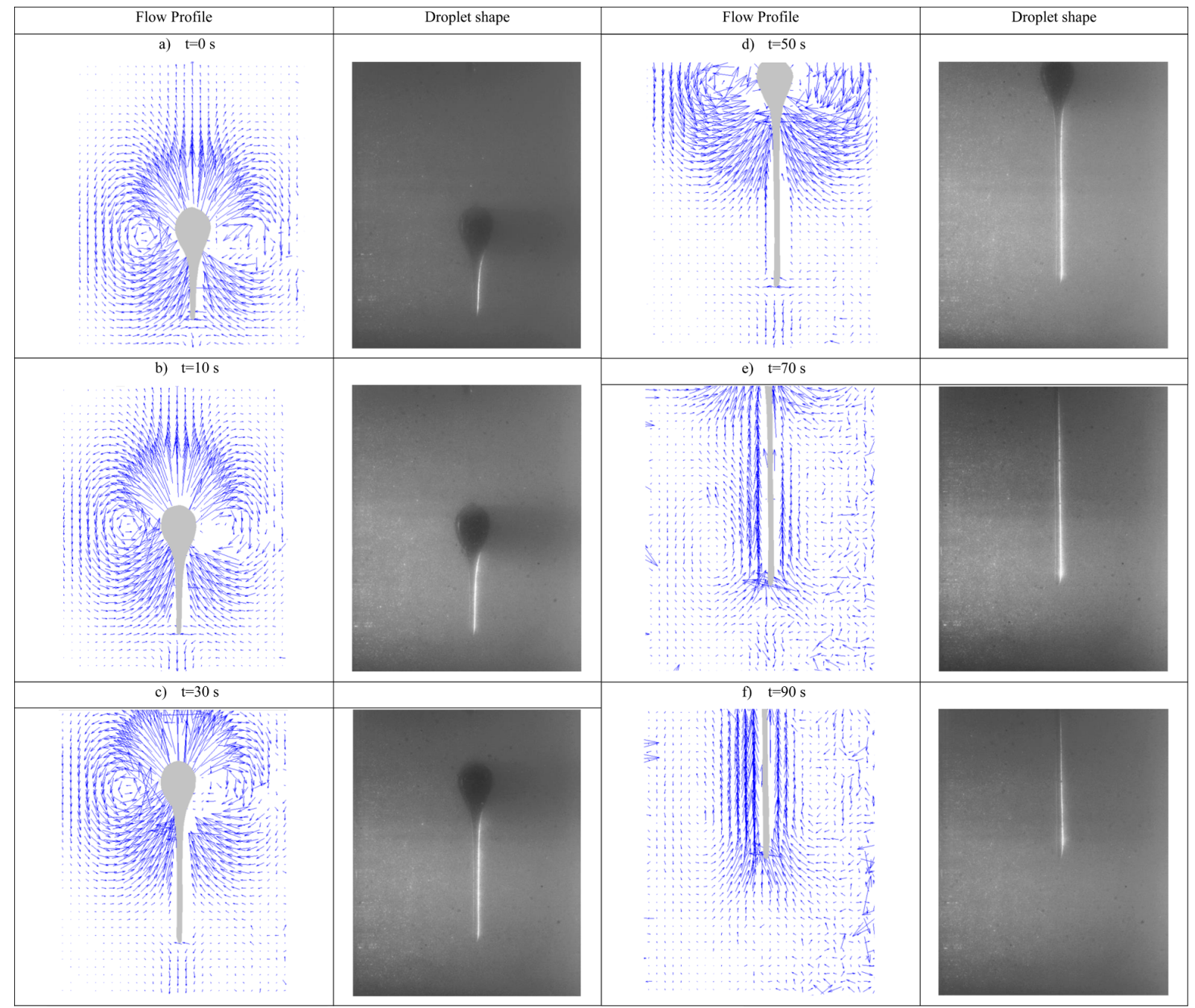

Fig. 9. (Color online) Time evolution of a viscoelastic droplet in a 5\% wt. Separan solution. 
In order to simplify the flow profile analysis, the droplet will be divided into two zones: Head and tail (Fig. 8). The flow around the head of the droplet is similar to that of a solid sphere falling through a viscoelastic fluid. A negative wake appears at the end of the tail.

\subsubsection{5\% wt Separan solution}

All the droplets injected through the 5\%wt Separan solution started with a tear-like shape, and after some time they adopted a more elongated shape. The flow profile for all the drops from $1 \mathrm{ml}$ to $5 \mathrm{ml}$ was similar. The velocity field and drop shape evolution in time is shown in Fig. 9.

At $t=0 \mathrm{~s}$ the drop has the typical flow profile of the tear-like shape bubbles and drops; A dipole-like flow around the drop's head, followed by a small negative wake. At $t=30 \mathrm{~s}$ the tail length grew a few centimeters. Note the presence of a negative wake closely behind the tip of the tail. The external liquid shows an upward flow on the sides of the tail. At $t=70 \mathrm{~s}$ the drop has stretched considerably, and the flow profile has started to change. The negative wake loses intensity while the tail keeps stretching. Finally the negative wake disappeared at $t=90$ s. Nevertheless, the drop kept stretching until breakup.

\subsection{Droplet breakup}

It was observed that Newtonian droplets (rising in nonNewtonian fluids) of sufficiently large volume form long tails that break at the very rear tip, leaving behind a trail of small, equally sized droplets. On the other hand, viscoelastic drops (when sufficiently large) break somewhere in between the tail, giving place to a couple of drops,

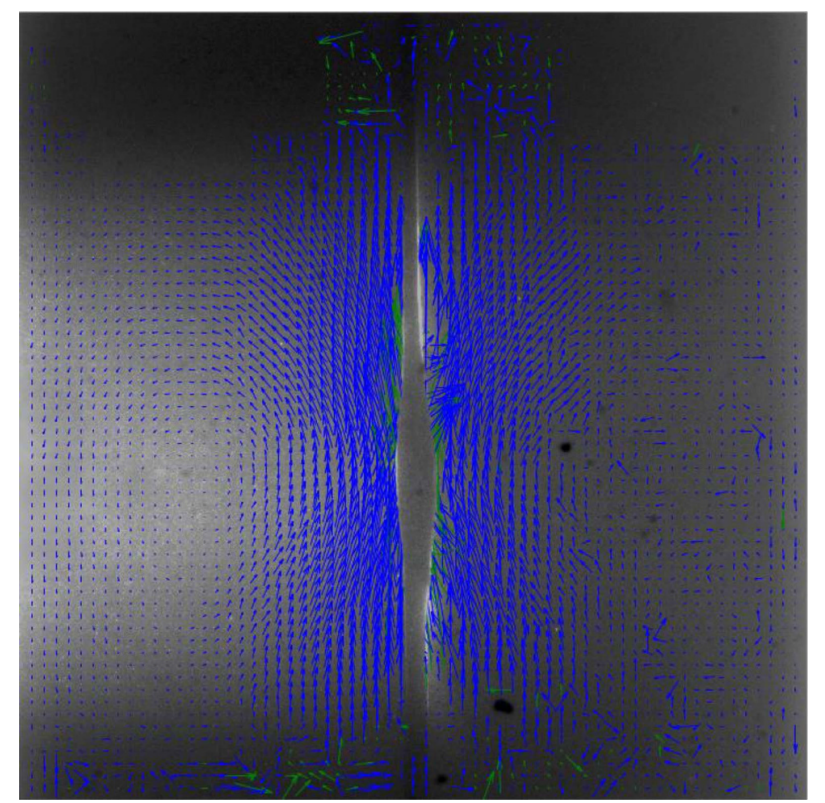

Fig. 10. (Color online) A bulge that formed on the tail of a $6 \mathrm{ml}$ viscoelastic (S1A 5\%) droplet rising in non-Newtonian fluid. Breakup occurs at the upper neck. This breakup mechanism required very long tails (and droplet volumes).

which in turn, if sufficiently large, begin to develop their own tail, successively breaking pairwise (if their volume is large enough). Small droplets adopt a tear-like shape and do not develop tails.

Other breakup mechanism was (seldomly) observed, with very large droplets: A bulge is formed at some point (or points, if the droplet is large enough) of the tail, and

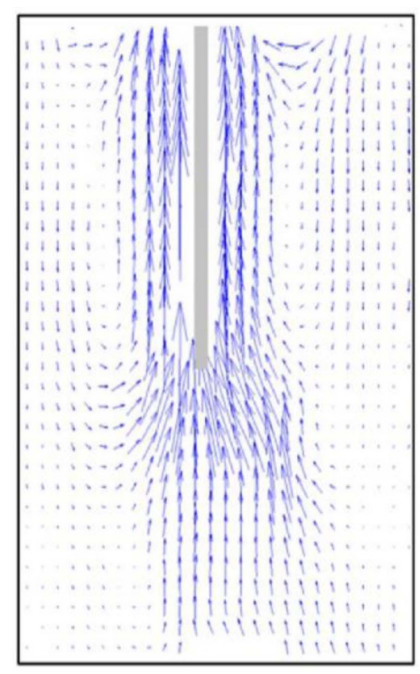

(a)

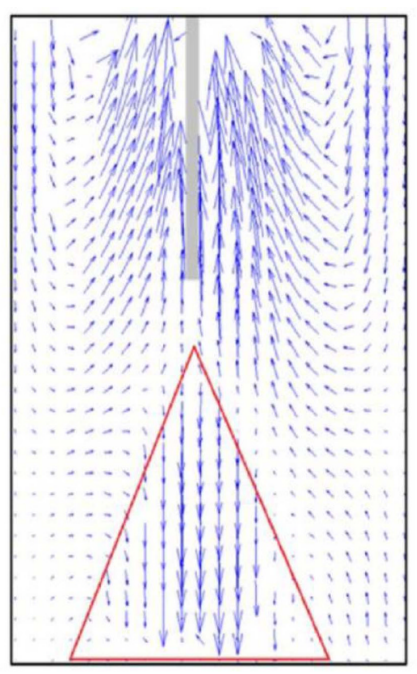

(b)

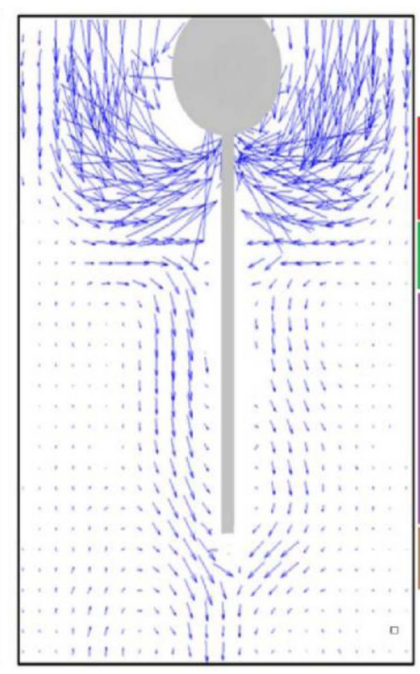

(c)

Fig. 11. (Color online) Flow around a solid droplet for three different types of external fluid: (a) Newtonian external fluid. An upward flow with no negative wake. (b) non-Newtonian 3\% solution (slightly viscoelastic); Note the appearance of the negative wake region (red zone) and (c) 5\% solution showing the appearance of the characteristic zones of the negative wake: Upstream zone (red zone) an stagnation zone where the flow reverses (green) and the downstream zone which affects the whole length of the tail and continuing downstream (purple and brown zone). 
breakup occurs at the upper neck. Figure 10 shows the onset of a viscoelastic droplet breakup of this type. It is clear from the figure that there exists a velocity gradient between the bulge's equator and the upper (and lower) neck, creating a flow pattern similar to that of a creeping flow around a spheroid.

In general, when the external fluid is non-Newtonian, the negative wake causes the formation of long tails, which develop under the effect of shear and elongational forces (Ortiz et al., 2016). As the tail gets thinner, elongational forces are subdued by shear forces and surface tension will induce breakup and formation of satellite droplets (Skelland and Raval, 1972; Kitamura and Takahashi, 1982; Clasen et al., 2006); once the radius of curvature is small enough, surface tension acts as the dominant force, causing necking and breakup of the tail.

\subsection{Comparison with a solid body}

In order to examine the droplet characteristics and the influence of the negative wake upon it, a solid body resembling the shape of the elongated droplet was introduced in the flow. The solid body consisted of a nylon sphere with a cylindrical shaft attached. The shape being similar to an elongated droplet but with no possibility of deforming; we may call it a solid droplet. The motion was then followed using the PIV technique.

The results are shown for the three different types of fluid. Figure 11 shows the position of the negative wake with respect to the droplet and its tail for the three types of fluid considered in the experiments:

a) External Newtonian fluid

b) External non-Newtonian fluid (3\% wt Separan Mixture)

c) External non-Newtonian fluid (5\% wt Separan Mixture)

It may be seen that the position of the negative wake relative to the body, depends on the elastic properties of the outer fluid. If the outer fluid is Newtonian, no negative wake will appear. However, as the elastic properties of the surrounding fluid are increased, the position of the negative wake will change accordingly. For the less viscoelastic fluid (Fig. 11b), the negative wake appears downstream after the tail whilst for the more viscoelastic fluid, it appears immediately after the head and acts upon the whole length of the tail. The flow along the tail is essentially an elongational flow and it acts in opposition to the shear stress caused by the tail being dragged in the direction of the motion of the droplet. This type of behavior was previously reported by Ortiz et al. (2016) for viscoelastic droplets with a long tail and is presently shown for solid droplets. So it may be inferred that it is not the droplet that determines the position or the intensity of the negative wake; this is strictly determined by the rheological characteristics of the surrounding fluid. However, if the

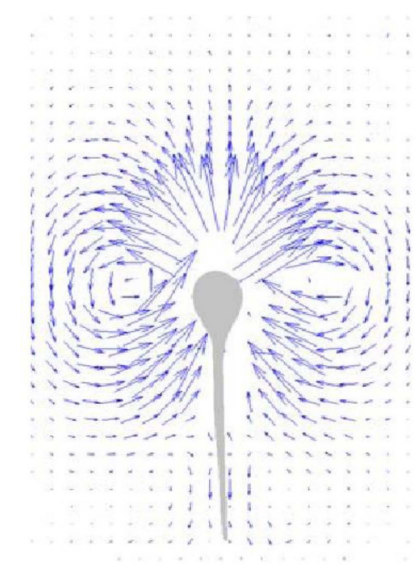

(a)

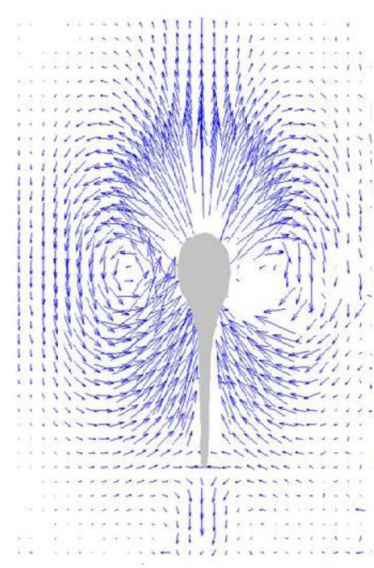

(b)
Fig. 12. (Color online) Flow field around $3 \mathrm{ml}$ (a) Newtonian drop and $3 \mathrm{ml}$ (b) non-Newtonian drop in 3\% wt Separan solution. In (a) the negative wake is around the tail of the droplet while in (b) the negative wake appears below the tail of the droplet.

droplet is viscoelastic and deformable in nature, the negative wake causes an elongational stress along the tail of the droplet and affects the type of breakup of the tail (Ortiz et al., 2016). In the case of the solid droplet, there is no possibility of elongation of the tail so the effect of the negative wake upon the flow is virtually negligible. This is not the case for a viscoelastic droplet where the deformation of the tail due to the elongational stresses competes against the shear stress along the tail. This will determine the type of tail breakup.

\section{Discussion}

All the Newtonian droplets injected through viscoelastic media presented a tail which breaks into droplets of different volumes and a downward flow on the sides of the tail (Fig. 12a). On the other hand, non-Newtonian droplets presented an upward flow on the sides of the tail and a downward flow (negative wake) below the tip of the tail (Fig. 12b) the droplets of $1 \mathrm{ml}$ of viscoelastic fluid presented a different behavior in the 3\%wt and 5\%wt Separan solution. The droplets injected in the 3\%wt Separan solution developed a tear like shape and kept this shape during the experiment. The drops injected in the 5\%wt Separan solution evolved from tear-like to elongated shape.

Both the $1 \mathrm{ml}$ droplets injected in the $3 \% \mathrm{wt}$ and $5 \% \mathrm{wt}$ Separan solution presented a negative wake. In Fig. 13, we show the flow profile for a $1 \mathrm{ml}$ drop traveling up in the 3\%wt Separan solution (Fig. 13a) and 5\%wt Separan solution (Fig. 13b). The flow profile for both drops is similar; a dipole-like flow around the head and a negative wake below the drop. However, the drops injected in the $5 \%$ wt Separan solution developed longer tails and the 


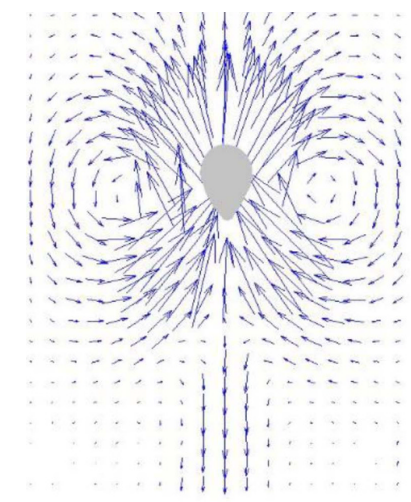

(a)

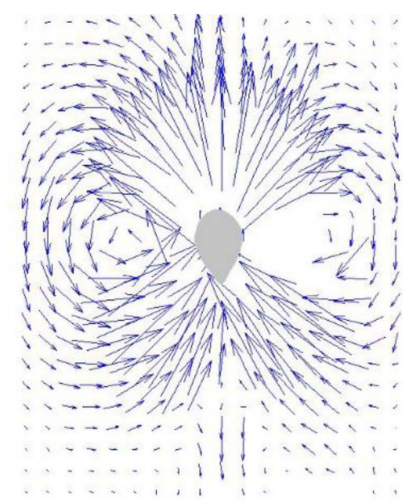

(b)
Fig. 13. (Color online) Flow field of two viscoelastic $1 \mathrm{ml}$ drops in (a) 3\% wt and (b) 5\% wt Separan Mixture. The final rising velocity of the drops are $U=1 \times 10^{-2} \mathrm{~m} / \mathrm{s}$ for (a) and $U=4.8 \times 10^{-}$ ${ }^{3} \mathrm{~m} / \mathrm{s}$ (b). Droplets rising through the $5 \%$ wt Separan mixture stretch until breakdown unlike the droplet in the 3\% Separan mixture, which keeps is initial shape all the way up.

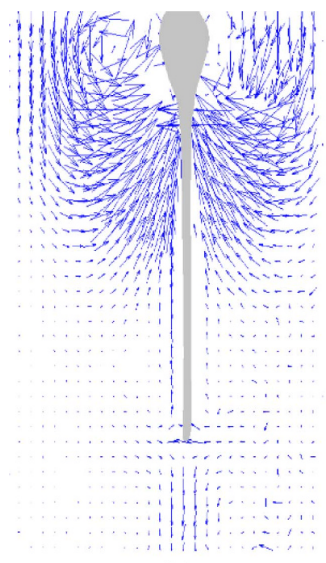

(a)

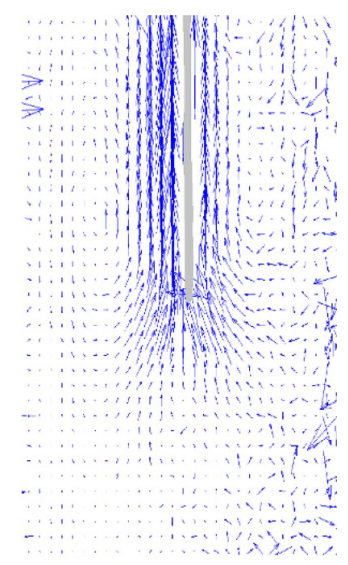

(b)
Fig. 14. (Color online) Flow field around $5 \mathrm{ml}$ viscoelastic drop in $5 \%$ wt Separan solution. The negative wake disappears after a while. Nevertheless, the drop keeps stretching until breakdown.

appearance of the negative wake occurred closer to the head.

Once the drop reached its final shape, the negative wake eventually disappeared. In Fig. 14 we show the time evolution of the flow profile for a $5 \mathrm{ml}$ droplet. From $\mathrm{t}=50$ $\mathrm{s}$ to $\mathrm{t}=90 \mathrm{~s}$ the negative wake became weaker until it disappeared. Once the negative wake disappeared the drop kept deforming until breakup.

\section{Conclusions}

These are some results that may be drawn from the experiments: The negative wake is a nonlinear elastic response associated with specific forms of the material functions and is only due to the viscoelastic properties of the external fluid. It may appear regardless of the nature of the body traveling through it. i.e. whether it is a solid, a bubble or a droplet. The largest velocities in the negative wake are concentrated behind the droplet in the region of extensional flow where the extensional viscosity is large. This wake structure is bounded by a region of high shear at the equator of the droplet where shear thinning lowers the viscosity. The influence of the negative wake, if any, upon the total drag of the body is very difficult to ascertain and to evaluate. In the case of viscoelastic droplets rising through a viscoelastic fluid when a tail appears, the negative wake may contribute to the elongational stress acting on the tail but this contribution appears to be very small compared to the competing shear stress acting along the tail. Nevertheless this contribution is important for the final breakup of the tail.

The negative wake is not caused by the droplet deformation; conversely, the presence of a negative wake does not imply the deformation of the (non-Newtonian) drops. The negative wake appears to be a consequence of the external flow viscoelastic properties only.

We found that Newtonian droplets rising through a nonNewtonian fluid show downwards flow (negative wake) on the sides of the tail while the viscoelastic drops show the negative wake behind the drop's tail.

The smaller drops of $1[\mathrm{ml}]$ injected through the $3 \% \mathrm{wt}$ Separan mixture show a negative wake in their flow profile. However, they do not suffer any deformation as they rise through a non-Newtonian external liquid.

Due to their physical properties, Newtonian fluids do not exhibit the negative wake phenomenon. Nevertheless, all the non-Newtonian droplets injected through a Newtonian fluid shifted from an initial tear-like shape to an elongated form with a long and resistant tail, similarly to the case of the viscoelastic drops when they rise through a non-Newtonian fluid except, for the latter case the break up system is different (Ortiz et al., 2016). For the appearance of a negative wake, the outer fluid must exhibit both shear thinning and elastic effects. The position of the negative wake will depend only on the external fluid viscoelastic properties, regardless of the nature of the droplet itself. However, if the latter is of a viscoelastic nature, elongational effects will affect the type of breakup.

\section{Acknowledgements}

The authors would like to thank CONACYT for Fernando Carril's support during his studies. Also, we acknowledge support from UNAM grants PAPIIT IN 106512, IT106018 and IT100218. D. Samayoa wishes to thank the Instituto Politécnico Nacional for the financial support during his sabbatical year at UNAM and B.Mena thanks support from PASPA program (DGAPA) during a sabbatical leave at the University of Seville. 
The influence of the negative wake on the deformation and breakup of viscoelastic droplets

\section{References}

Arigo, M.T. and G.H. Mckinley, 1998, An experimental investigation of negative wakes behind spheres settling in a shearthinning viscoelastic fluid, Rheol. Acta 37, 307-327.

Bisgaard, C., 1983, Velocity fields around spheres and bubbles investigated by laser-doppler anemometry, J. Non-Newton. Fluid Mech. 12, 283-302.

Brinson, H.F. and L.C. Brinson, 2008, Polymer Engineering Science and Viscoelasticity: An Introduction, Springer, Boston.

Broadbent, J. and B. Mena, 1974, Slow flow of an elastico-viscous fluid past cylinders and spheres, Chem. Eng. J. 8, 11-19.

Bush, M.B., 1993, The stagnation flow behind a sphere, J. NonNewton. Fluid Mech. 49, 103-122.

Campo-Deaño, L., R.P.A. Dullens, D.G.A.L. Aarts, F.T. Pinho, and M.S.N. Oliveira, 2013, Viscoelasticity of blood and viscoelastic blood analogues for use in polydymethylsiloxane in vitro models of the circulatory system, Biomicrofluidics 7, 034102.

Carril, F., 2019, Influencia de la estela negativa en la deformacin $y$ ruptura de gotas viscoelasticas, M.S. Thesis, National Autonomous University of Mexico.

Caswell, B., O. Manero, and B. Mena, 2004, Recent developments on the slow viscoelastic flow past spheres and bubbles, Rheol. Rev. 2004, 197-223.

Cherdhirankorn, T., W. Lerdwijitjarud, A. Sirivat, and R.G. Larson, 2004, Dynamics of vorticity stretching and breakup of isolated viscoelastic droplets in an immiscible viscoelastic matrix, Rheol. Acta 43, 246-256.

Clasen, C., J. Eggers, M.A. Fontelos, J. Li, and G.H. McKinley, 2006, The beads-on-string structure of viscoelastic threads, $J$. Fluid Mech. 556, 283-308.

Dou, H.S. and N. Phan-Thien, 2003, Negative wake in the uniform flow past a cylinder, Rheol. Acta 42, 383-409.

Fraggedakis, D., M. Pavlidis, Y. Dimakopoulos, and J. Tsamopoulos, 2016, On the velocity discontinuity at a criticalvolume of a bubble rising in a viscoelastic fluid, J. Fluid Mech. 789, 310-346.

Frank, X. and H.Z. Li, 2006, Negative wake behind a sphere rising in viscoelastic fluids: A lattice Boltzmann investigation, Phys. Rev. E 74, 056307.

Harlen, O.G., 2002, The negative wake behind a sphere sedimenting through a viscoelastic fluid, J. Non-Newton. Fluid Mech. 108, 411-430.

Hassager, O., 1979, Negative wake behind bubbles in non-newtonian liquids, Nature 279, 402-403.

Hou, J., Z. Liu, S. Zhang, X. Yue, and J. Yang, 2005, The role of viscoelasticity of alkali/surfactant/polymer solutions in enhanced oil recovery, J. Pet. Sci. Eng. 47, 219-235.

Imaizumi, Y., T. Kunugi, T. Yokomine, and Z. Kawara, 2014, Viscoelastic fluid behaviors around a rising bubble via a new method of mesh deformation tracking, Chem. Eng. Sci. 120, 167-173.

Joseph, D.D. and J. Feng, 1995, The negative wake in a secondorder fluid, J. Non-Newton. Fluid Mech. 57, 313-320.

Kemiha, M., X. Frank, S. Poncin, and H.Z. Li, 2006, Origin of the negative wake behind a bubble rising in non-Newtonian fluids, Chem. Eng. Sci. 61, 4041-4047.

Kitamura, Y. and T. Takahashi, 1982, Breakup of jets in power law non-newtonianndashnewtonian liquid systems, Can. J. Chem. Eng. 60, 732-737.

Manero, O. and B. Mena, 1981, On the slow flow of viscoelastic liquids past a circular cylinder, J. Non-Newton. Fluid Mech. 9, 379-387.

Matsumura, Y., I.J. Kang, H. Sakamoto, M. Motoki, and T. Mori, 1993, Filler effects of oil droplets on the viscoelastic properties of emulsion gels, Food Hydrocolloids 7, 227-240.

Mena, B., O. Manero, and L.G. Leal, 1987, The influence of rheological properties on the slow flow past spheres, J. Non-Newton. Fluid Mech. 26, 247-275.

Mendoza-Fuentes, A.J., R. Montiel, R. Zenit, and O. Manero, 2009 , On the flow of associative polymers past a sphere: Evaluation of negative wake criteria, Phys. Fluids 21, 033104.

Milliken, W.J. and L.G. Leal, 1991, Deformation and breakup of viscoelastic drops in planar extensional flows, J. Non-Newton. Fluid Mech. 40, 355-379.

Mukherjee, S. and K. Sarkar, 2009, Effects of viscosity ratio on deformation of a viscoelastic drop in a Newtonian matrix under steady shear, J. Non-Newton. Fluid Mech. 160, 104-112.

Ortiz, S.L., J.S. Lee, B. Figueroa-Espinoza, and B. Mena, 2016, An experimental note on the deformation and breakup of viscoelastic droplets rising in non-Newtonian fluids, Rheol. Acta 55, 879-887.

Plog, J., J. Wu, Y.J. Dias, F. Mashayek, L.F. Cooper, and A.L. Yarin, 2020, Reopening dentistry after COVID-19: Complete suppression of aerosolization in dental procedures by viscoelastic Medusa Gorgo, Phys. Fluids 32, 083111.

Satrape, J.V. and M.J. Crochet, 1994, Numerical simulation of the motion of a sphere in a Boger fluid, J. Non-Newton. Fluid Mech. 55, 91-111.

Skelland, A.H.P. and V.K. Raval, 1972, Drop size in power law non-Newtonian systems, Can. J. Chem. Eng. 50, 41-44.

Soto, E., C.G. Goujon, R. Zenit, and O. Manero, 2006, A study of velocity discontinuity for single air bubbles rising in an associative polymer, Phys. Fluids 18, 121510.

Suhr, J., N. Koratkar, P. Keblinski, and P. Ajayan, 2005, Viscoelasticity in carbon nanotube composites, Nat. Mater. 4, 134137.

Zenit, R. and J.J. Feng, 2018, Hydrodynamic interactions among bubbles, drops, and particles in non-Newtonian liquids, Annu. Rev. Fluid Mech. 50, 505-534.

\section{Publisher's Note}

Springer Nature remains neutral with regard to jurisdictional claims in published maps and institutional affiliations. 